\title{
Image Processing of HRTEM images of Hydroxyapatite
}

S.Tehuacanero*, A.Lopéz, J.Reyes-Gasga.

Instituto de Física, Universidad Nacional Autónoma de México, Apdo. Postal 20-364, 01000

México, D. F., México.

Digital image processing techniques are used to study of the Hydroxyapatite images under high resolution HRTEM condition. This is a very important material in the biomaterial field, it is due to it's excellent biocompatibility. Nowadays it is widely spread in dentistry treatments and in human medicine and veterinary.

In recent years in characterization of the hydroxyapatite material by HRTEM, it has become clear that the matching of high resolution transmission electron microscopy with image processing is absolutely essential to the understanding and correct interpretation of its images. This commonly present constrast of no easy interpretation, associated to noise contribution that may be have various origins: a carbon support film can give rise to interfering constrast effects, residual amorphous layer due to preparation techniques can be present on the specimen; during electron irradiation, radiation damage and inelastic scattering can further obscure the relevant structural information in the HRTEM image[1].

In the present work we show the results of the application of two segmentation processes of images. The first called k-mean, the second Bayes funtion and technical of Fourier filtering [2] in hydroxyapatite HRTEM images where the objetive is to classify the structures in the hidroxiapatite image and with this to have a better understanding and interpretation.

These images were obtained using a Jeol-4000FX microscopy. Image processing was made using a CCD high resolution camera to processed the film plates. Image calculation were perfomed using programs developed in Matlab.

In figure 1 we show the original image obtained by HRTEM. For the enhancement the contrast of this image a window with the Hamming filter of frecuency 0.25 and 0.65 (fig.2) was used. The image processed is showing in figure 3 . Now, the structures are inset into the image, found the identification of structures in the image with the Bayes function algorithm (fig.5) give us a better result, with respect to the k-mean algorithm (fig.4). The areas damaged were identified, and easily observed from the structures inset of the areas. Finally, we would like to comment that our results are of general application for a better interpretation of the information contained in the images of HRTEM of hydroxyapatite.

\section{References}

[1] J. Reyes-Gasga and R. García-García, Radiation Phys. and Chem. 64(2002)359-367).

[2] John C.Russ. The Image Processing.Handbook.CRC1992.

[ 3] We want to thank the technical help from L.Beltran, R.Hernández, L.Rendón, P.Mexia, J.Cañetas, C. Magaña, C.Flores, A.Sánchez. 


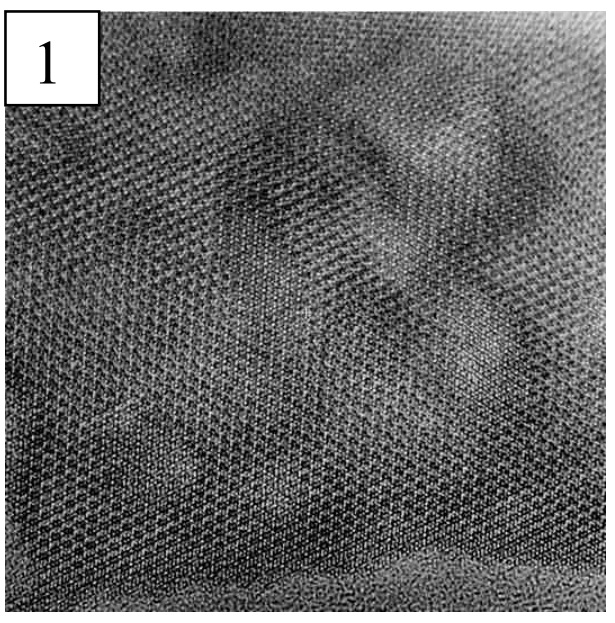

Fig.1.Original Image of hydroxyapatite obtained by HRTEM.
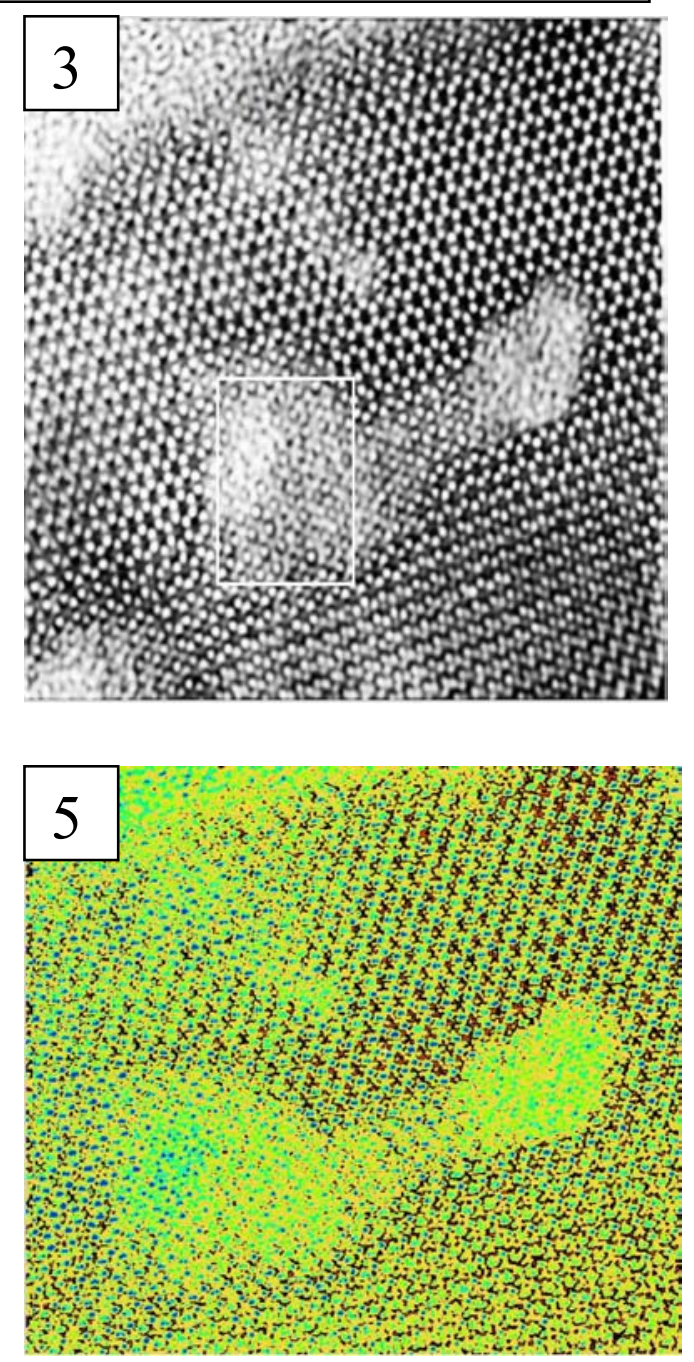

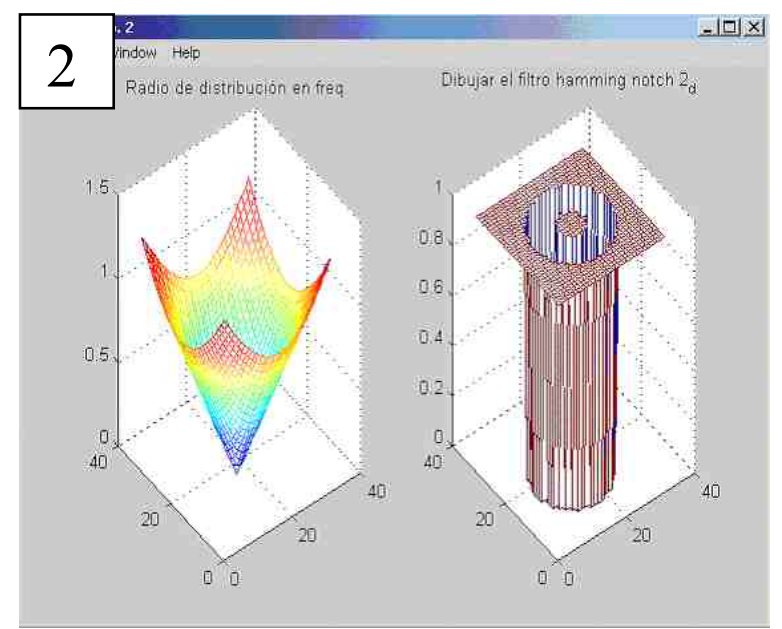

Fig.2. Hamming filter with frecuency of 0.25 and 0.65 .

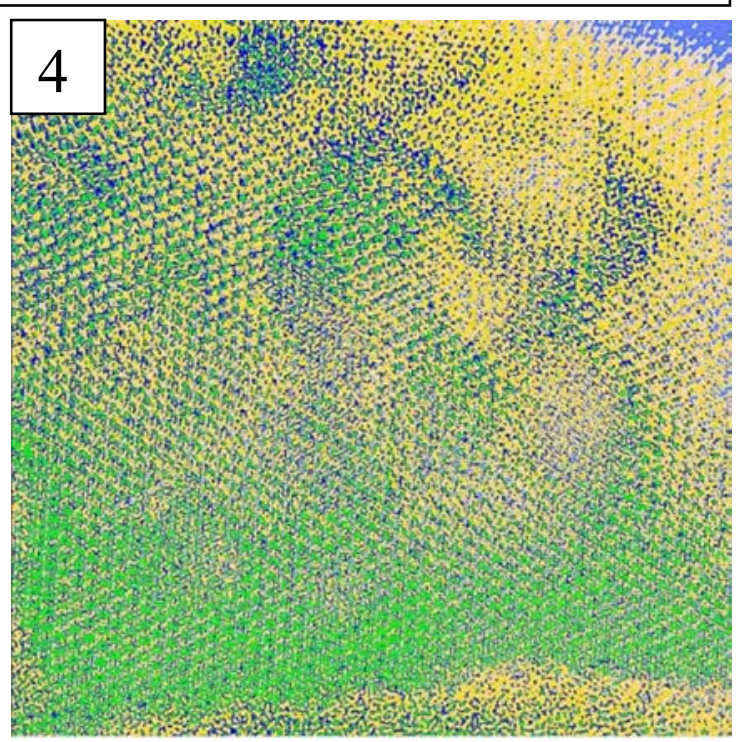

Fig.3.Image processig of HRTEM of image hydroxyapatite with Hamming filter.

Fig.4. Image processed with K-mean algoritm. This procedure recognizes a number of Clusters: 6

Fig.5. Clasified image with Bayes function algorithm. 